\title{
The $\mathrm{U}_{\mathrm{L}} 21$ Gene Products of Herpes Simplex Virus 1 Are Dispensable for Growth in Cultured Cells
}

\author{
JOEL D. BAINES,${ }^{1} \dagger$ A. HAJIME KOYAMA, ${ }^{1} \ddagger$ TIANMIN HUANG, ${ }^{2}$ AND BERNARD ROIZMAN ${ }^{1 *}$ \\ The Marjorie B. Kovler Viral Oncology Laboratories, The University of Chicago, Chicago, Illinois 60637, ${ }^{1}$ and \\ Section of Microbiology and Virology, Department of Experimental Pathology, \\ University of Bologna, Bologna, Italy ${ }^{2}$
}

Received 27 October 1993/Accepted 20 January 1994

\begin{abstract}
A viral deletion mutant $\left(\Delta U_{L} 21\right)$ that lacked the sequences encoding 484 of the predicted first 535 amino acids of the $U_{L} 21$ open reading frame was genetically engineered and studied with respect to its phenotype in cells in culture. We report the following. (i) The replication of $\Delta U_{L} 21$ was identical to that of the parent herpes simplex virus 1 (HSV-1) strain $F$ in Vero cells, but the yields were three- to fivefold lower than those of the parent virus in human embryonic lung cells. (ii) To characterize the $U_{L} 21$ protein, we immunized rabbits against a purified bacterial fusion protein consisting of glutathione $S$-transferase fused to the majority of the coding domain of the $U_{L} 21$ gene. Rabbit antiserum directed against the fusion protein recognized a broad band with an apparent $M_{\mathrm{r}}$ of 62,000 to 64,000 in lysates of cells infected with HSV-1 strain $F$ and in virions purified from the infected cell cytoplasm. This band was absent from lysates of mock-infected cells or cells infected with the $\Delta U_{L} 21$ virus. The band was significantly reduced in intensity in lysates of cells infected in the presence of phosphonoacetic acid, indicating that it is expressed as a late $(\gamma 1)$ gene. (iii) Immunofluorescence studies localized the $U_{L} 21$ antigen primarily in brightly staining granules in the cytoplasms of infected cells. Taken together, the data indicate that the $U_{L} 21$ protein is a virion component dispensable for all aspects of replication of HSV-1 in the cells tested. The electrophoretic mobility of the $U_{L} 21$ protein suggests that it is extensively modified posttranslationally.
\end{abstract}

The herpes simplex virus 1 (HSV-1) genome consists of two covalently linked components, $\mathrm{L}$ and $\mathrm{S}$. The $\mathrm{L}$ and $\mathrm{S}$ components each consist of unique sequences, $U_{L}$ and $U_{S}$, respectively, flanked by inverted repeats $(17,34,38)$. The genome of HSV-1 encodes at least 77 genes $(1,6,15,19,23-25)$. Of the genes known to be encoded by the HSV genome, 58 map in the $U_{L}, 13$ map in the $U_{S}$, and two copies of 3 genes map in the reiterated sequences which flank $U_{L}$ and $U_{S}$. Of the genes encoded in $U_{L}$, at least 23 have been shown to be dispensable for replication in cells in culture. Thus, the $\mathrm{U}_{\mathrm{L}} 2, \mathrm{U}_{\mathrm{L}} 3, \mathrm{U}_{\mathrm{L}} 4$, $\mathrm{U}_{\mathrm{L}} 10, \mathrm{U}_{\mathrm{L}} 11, \mathrm{U}_{\mathrm{L}} 12, \mathrm{U}_{\mathrm{L}} 13, \mathrm{U}_{\mathrm{L}} 16, \mathrm{U}_{\mathrm{L}} 20, \mathrm{U}_{\mathrm{L}} 23, \mathrm{U}_{\mathrm{L}} 24, \mathrm{U}_{\mathrm{L}} 39$, $\mathrm{U}_{\mathrm{L}} 40, \mathrm{U}_{\mathrm{L}} 41, \mathrm{U}_{\mathrm{L}} 43, \mathrm{U}_{\mathrm{L}} 44, \mathrm{U}_{\mathrm{L}} 45, \mathrm{U}_{\mathrm{L}} 46, \mathrm{U}_{\mathrm{L}} 47, \mathrm{U}_{\mathrm{L}} 50, \mathrm{U}_{\mathrm{L}} 51$, $\mathrm{U}_{\mathrm{L}} 55$, and $\mathrm{U}_{\mathrm{L}} 56$ genes are dispensable for replication in some cultured cell lines $(2,3,5,7,13,14,16,18,21,22,27-32,37,40$, 42).

In this article, we report on the construction and properties of a new viral deletion mutant solely lacking the $U_{L} 21$ open reading frame. Interest in this open reading frame stems from a recent report that a homolog of $U_{L} 21$ in pseudorabies virus (PRV) is a component of the capsid involved in DNA cleavage and packaging (11). We report that the HSV-1 $U_{L} 21$ protein is not essential for viral replication. In addition, we describe some of the properties of the protein encoded by the $\mathrm{U}_{\mathrm{L}} 21$ gene.

\footnotetext{
* Corresponding author. Mailing address: The Marjorie B. Kovler Viral Oncology Laboratories, The University of Chicago, 910 E. 58th St., Chicago, IL 60637. Phone: (312) 702-1898. Fax: (312) 702-1631.

$\dagger$ Present address: Schurman Hall, Cornell University, Ithaca, NY 14853.

$\ddagger$ Present address: Department of Virology, School of Medicine, The University of Tokushima, Kuramoto 3, Tokushima 770, Japan.
}

\section{MATERIALS AND METHODS}

Cells and viruses. Viruses were titered in Vero cells. The studies on viral proteins were done on lysates of infected HEp-2 cells. All cell lines were maintained in Dulbecco's modified Eagle medium supplemented with $5 \%$ newborn calf serum. After infection, the cells were maintained in mixture 199 medium supplemented with $1 \%$ newborn calf serum. Titrations and preparations of viral stocks were done as described previously (2).

HSV-1 strain F [HSV-1(F)] is a limited-passage strain used as the HSV-1 prototype in this laboratory (12). HSV-1(F) $\Delta 305$ is a recombinant virus from which $500 \mathrm{bp}$ had been deleted in the thymidine kinase $(t k)$ gene (29). Recombinant viruses described in this report were derived from HSV-1(F) $\Delta 305$. The recombinant virus $\mathrm{R} 7214$, containing a $t k$ gene inserted between the $U_{L} 20$ and $U_{L} 21$ open reading frames, has been described previously (5). The recombinant R7202, containing a lac $Z$ gene inserted in place of a large fraction of the sequences encoding glycoprotein $\mathrm{E}$, has also been described (4).

Reagents and plasmids. Restriction enzymes were obtained from commercial sources as previously described (2). The plasmid pRB451 contains a HindIII-EcoRI fragment from HSV-1(F) DNA cloned into the vector pGEM3Z (Promega) as previously described (5). This DNA fragment contains the entire $U_{L} 20$ and $U_{L} 21$ open reading frames within the Bam $H I$ $\mathrm{F}^{\prime}$ and $\mathrm{BamHI} \mathrm{T}$ fragments of HSV-1 DNA, respectively (see Fig. 1). To delete the $U_{L} 21$ gene, pRB451 was digested with $B s t E I I$ and religated. The deleted BstEII fragment contained a total of $1,812 \mathrm{bp}$ and included the DNA sequences encoding the N-terminal 484 codons of the total of 535 codons encoded by the $U_{L} 21$ open reading frame. This plasmid was designated pRB4129.

A DNA oligomer (A AAG GGC CAG CAG AAG CCC 
AAC CTG CTG GAC CGC CTG CGC CAC CGC AAG AAC GGG TAC CGC CAC GA, and its complement) encoding the epitope N-KGQKPNLLDRLRHRKNGYRNH-C, which is recognized by the mouse monoclonal antibody CH28-2 reactive with a human cytomegalovirus (CMV) epitope in glycoprotein B (8), was cloned into an NruI site between codons 399 and 400 of the $U_{L} 21$ open reading frame in the following arrangement: Arg (399)-epitope-Asp-Asp (400), where the aspartic acid codon was added to maintain the proper reading frame. The DNA contained in this segment of the genome was sequenced for verification. The plasmid containing the tagged $\mathrm{U}_{\mathrm{L}} 21$ open reading frame was digested with HincII and EcoRI and was cloned into the SmaI-EcoRI sites of the bacterial fusion vector pGEX3X (Pharmacia) in frame with the gene encoding glutathione $S$-transferase (GST). In the final product, the DNA encoding the C-terminal 381 amino acids of the $U_{L} 21$ open reading frame including the inserted CMV epitope was fused to the gene encoding GST.

Selection of recombinant viruses. Human $143 \mathrm{TK}^{-}$cells were maintained in Dulbecco modified Eagle medium supplemented with 5\% newborn calf serum. Cells were infected and subsequently maintained under a hypoxanthine-aminopterinthymidine (HAT) or bromodeoxyuridine (BUdR; $50 \mu \mathrm{g} / \mathrm{ml}$ ) overlay, for selection of $t k^{-}$and $t k^{+}$recombinant viruses, respectively.

Purification and analysis of viral DNA. Viral DNA intended for transfections was prepared from NaI gradients (39). Otherwise, viral DNA was purified by phenol-chloroform extraction of cytoplasmic extracts of infected Vero cells. DNA probes were prepared by nick translation of 200 to $400 \mathrm{ng}$ of DNA according to the directions of the manufacturer of a commercially available kit (New England Nuclear). DNA was separated on $1.0 \%$ agarose gels and was transferred to two nitrocellulose sheets as previously described $(20,35)$. Conditions for hybridization with radiolabeled probes and washing of the hybridized sheets were as previously described (4).

Production of rabbit polyclonal antiserum. The $U_{\mathrm{L}} 21-\mathrm{GST}$ fusion protein was not soluble in 0.1 to $2.0 \%$ Triton X-100 but was soluble in $0.1 \%$ sodium dodecyl sulfate (SDS). Therefore, the fusion protein was purified from bacterial cell lysates by separation on a denaturing polyacrylamide gel. The gel was then stained with Coomassie brilliant blue, and the band of interest was electroeluted into a minimal volume of buffer containing $0.1 \%$ SDS, $50 \mathrm{mM}$ Tris- $\mathrm{HCl}(\mathrm{pH} \mathrm{7.4)}$ ), and $1 \mathrm{mM}$ EDTA. A female New Zealand White rabbit was immunized first with $250 \mu \mathrm{g}$ of this gel-purified fusion protein emulsified in complete Freund's adjuvant and subsequently four times with $250 \mu \mathrm{g}$ of the protein emulsified in Freund's incomplete adjuvant.

Immunoblotting and fluorescent antibody analyses. Immunoblotting and fluorescent antibody analyses were done essentially as previously described (4), except that the rabbit polyclonal antiserum was diluted 1:100 for fluorescence, 1:1,000 for staining electrophoretically separated, infected cell lysates electrically transferred to a nitrocellulose sheet, and 1:10,000 for staining immunoblots of purified virions.

Virion purification. Virions were purified from homogenates of infected HEp-2 cells as described by Spear and Roizman with minor modification as described by Baines and Roizman $(4,36)$.

\section{RESULTS}

Construction of a $\mathbf{U}_{\mathbf{L}} 21$ viral deletion mutant. The purpose of this series of experiments was to determine whether the $\mathrm{U}_{\mathrm{L}} 21$ gene was dispensable for viral replication in cells in culture. The general method by which this laboratory produces recombinant viruses has been described previously (30). Briefly, a selectable marker is placed at or near the target site in the viral genome by cotransfection of HSV-1(F) $\Delta 305$, a $t k^{-}$ virus, with a plasmid containing a $t k$ gene driven by the $\alpha 27$ gene promoter $(\alpha 27-t k)$ and inserted into the target sequence. The progeny of the transfection is then plated on $143 \mathrm{TK}^{-}$cells in HAT medium to enable only $t k^{+}$viruses to replicate. The DNA of this virus is then transfected with a plasmid from which the target sequences including the site of insertion of the $\alpha 27-t k$ gene had been deleted. The progeny of this transfection is then plated in $143 \mathrm{TK}^{-}$cells in the presence of BUdR. If the gene is dispensable for viral replication, then both of the recombinant viruses containing the inactivated gene by insertional mutagenesis or the deletion are readily obtained. In the final step, the truncated $t k$ gene is repaired by cotransfection of mutant virus DNA with DNA containing an intact natural $t k$ gene. Although mutants obtained in this fashion reflect solely the deletion, substitution, or insertion contained in the plasmid used in the transfection, we usually restore the deleted sequences by transfection with an appropriate fragment.

For the construction of a $U_{\mathrm{L}} 21$ deletion mutant, we took advantage of the fact that the genome of a previously constructed recombinant virus, R7214 (5), which was derived from the $t k^{-}$mutant HSV-1(F) $\Delta 305$, contained the $\alpha 27-t k$ gene between the $U_{L} 20$ and $U_{L} 21$ open reading frames (Fig. 1, lines 1 to 3). To make the $U_{\mathrm{L}} 21^{-}$virus, we constructed the plasmid pRB4129, from which the start codon and all but 51 of the predicted 535 codons of the $\mathrm{U}_{\mathrm{L}} 21$ open reading frame had been deleted. The construction of pRB4129 is described in Materials and Methods, and a diagram of the plasmid is shown in Fig. 1, line 4. R7214 viral DNA was cotransfected with pRB4129 plasmid DNA and $t k^{-}$progeny were selected from among the progeny of the cotransfection under a BUdR overlay. The $t k^{-}$progeny were plaque purified twice under a BUdR overlay, and the virus harvested individually from 12 plaques was used to infect Vero cells. Analyses of the viral DNA from the cytoplasm of infected Vero cells indicated the presence of the $U_{L} 21$ deletion in 10 of the 12 recombinant viral genomes (not shown). One of these viruses was plaque purified an additional two times in Vero cells and was designated R7222.

In order to rescue the natural $t k$ gene in R7222, rabbit skin cells were transfected with a plasmid DNA containing the BamHI Q fragment. This fragment contains the entire HSV-1 $t k$ gene driven by its natural promoter. The transfected cells were then infected with the R7222 virus. The progeny of the infection was plated on $143 \mathrm{TK}^{-}$cells in HAT medium, and a $t k^{+}$virus, designated $\mathrm{R} 7228$, was plaque purified. Finally, R7228 viral DNA was cotransfected with purified pRB451 DNA containing an intact $U_{L} 21$ gene (5). The progeny of the transfection was plated on Vero cells, and large plaques were harvested, plaque purified, and screened for the presence of viral progeny containing an intact $\mathrm{U}_{\mathrm{L}} 21$ gene. The plaquepurified, marker-rescued virus was designated R7242. No large plaques were evident in an experiment performed in parallel in which R7228 DNA was cotransfected with pGEM3Z DNA (not shown), suggesting that the large plaques present among the progeny of transfection of R7242 DNA with the pRB451 reflected the $\mathrm{U}_{\mathrm{L}} 21^{+}$phenotype.

Figure 2 illustrates the changes in the electrophoretic mobility of the relevant DNA fragments of the wild-type and of recombinant viruses made in this and previous studies. Specifically, Bam HI digests of purified HSV-1(F), HSV-1(F) $\Delta 305$, R7214, R7228, and R7242 DNAs were electrophoretically separated on a $1.0 \%$ agarose gel, transferred to two sheets of 

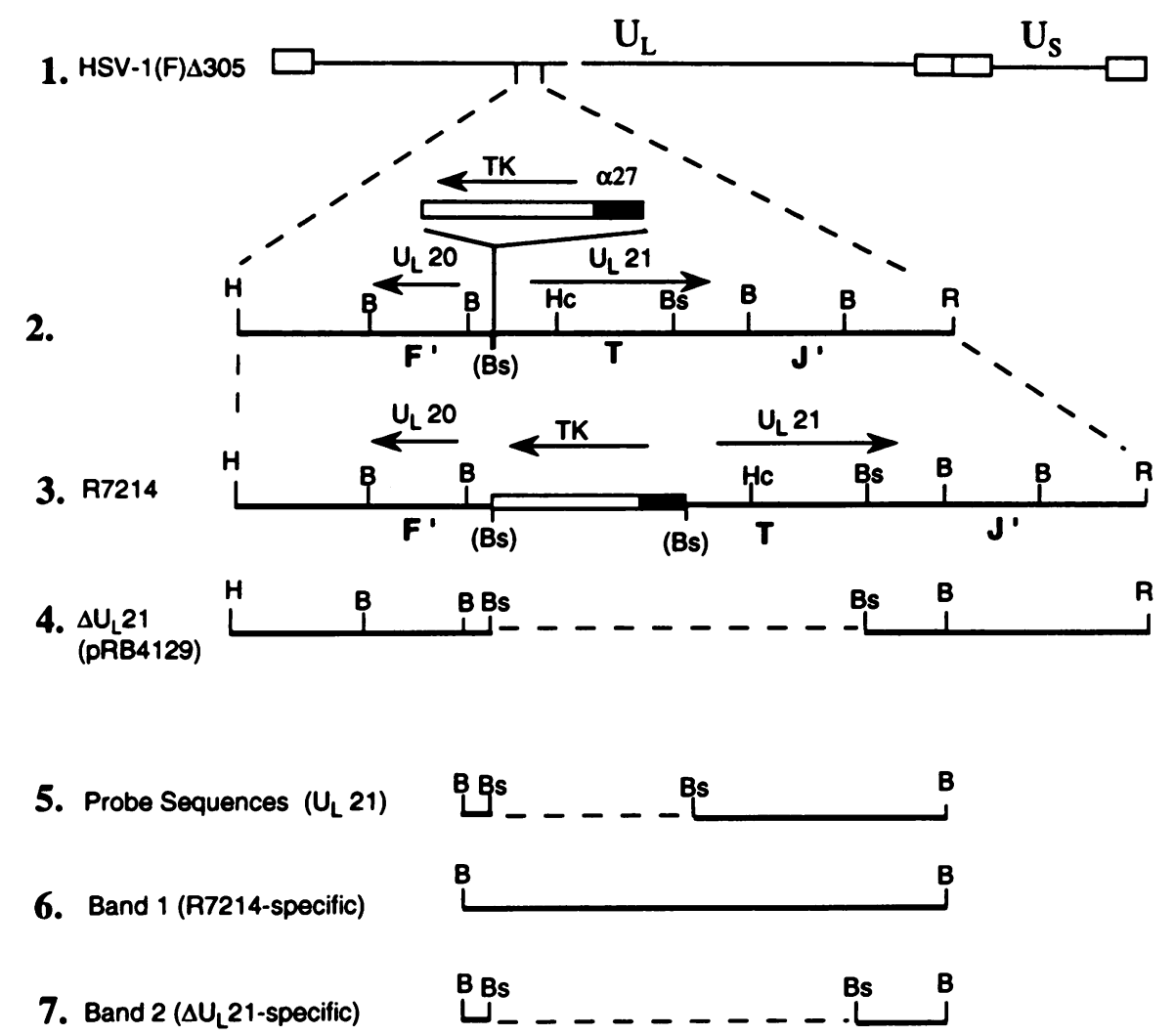

FIG. 1. Schematic colinear representation of the sequence arrangements of HSV-1 plasmid and viral DNAs relevant to these studies. Line 1, schematic representation of HSV-1(F) $\Delta 305$ DNA. Open rectangles represent inverted repeats flanking the unique sequences. The deletion of the natural $t k$ gene of this virus is represented by a gap within the line representing the unique long $\left(\mathrm{U}_{\mathrm{L}}\right)$ genomic component. Lines 2 and 3 , schematic representation of the construction of the recombinant virus R7214 containing an insertion of the $t k$ gene (open rectangle) driven by the $\alpha 27$ promoter (filled rectangle) between the $U_{L} 20$ and $U_{L} 21$ open reading frames of HSV-1(F) $\Delta 305$. Arrows indicate the direction and length of the respective open reading frames. The position of the Bam $\mathrm{HI} \mathrm{F}^{\prime}, \mathrm{T}$, and $\mathrm{J}^{\prime}$ fragments described in experiments whose results are illustrated in Fig. 2 are indicated below line 3. Line 4, schematic representation of HSV-1(F) sequences within the plasmid pRB4129 used to produce the recombinant virus $R 7228$ lacking the majority of the $U_{L} 21$ open reading frame. The dashed line represents a deletion of the chimeric $t k$ gene and the $U_{L} 21$ open reading frame. Line 5, schematic representation of the Bam HI T probe. The probe does not contain $t k$ sequences; the deleted sequences are indicated by a dashed line. Line 6, schematic representation of sequence arrangement of band 1 specific for R7214 DNA. This $B a m \mathrm{HI}$ band arose as a consequence of the insertion of the $\alpha 27-t k$ gene within the Bam HI T fragment of R7214 and contains sequences homologous to the probe represented in line 5. Line 7, representation of band 2 specific for R7228 DNA. The deletion of the $U_{L} 21$ and chimeric $t k$ genes (dashed line) causes the Bam HI T fragment in R7228 $\left(\Delta \mathrm{U}_{\mathrm{L}} 21\right)$ viral DNA to be severely truncated. B, Bam HI; Bs, BstEII; H, HindIII; Hc, HincII; R, EcoRI. Restriction sites in parentheses were destroyed during cloning.

nitrocellulose, and probed, respectively, with labeled Bam HI T fragment which contains $\mathrm{U}_{\mathrm{L}} 21$ sequences and with the Bam $\mathrm{HI}$ $\mathrm{Q}$ fragment containing the $t k$ gene. The results were as follows.

(i) The Bam HI T fragments of HSV-1(F) and HSV$1(F) \Delta 305$ containing the $U_{L} 21$ open reading frame (Fig. 2) could not be differentiated with respect to electrophoretic mobility. They migrated at a position consistent with a size of approximately $2.7 \mathrm{kbp}$.

(ii) The fragment hybridizing with $B a m H I$ T sequences of R7214 DNA migrated at a position consistent with a size of 4.5 kbp (Fig. 2, band 1). Thus, it was significantly larger than the corresponding HSV-1(F) and HSV-1(F) $\Delta 305$ fragments. A schematic diagram of the predicted sequence arrangement in band 1 is shown in Fig. 1, line 6. Since this band also hybridized with the $t k$ probe (Fig. 2, lane 8), the increase in size is due to the insertion of the chimeric $\alpha 27-t k$ gene.

(iii) The BamHI Q fragment of HSV-1(F) migrated more slowly than the corresponding HSV-1(F) $\Delta 305$ and R7214 DNA fragments (Fig. 2, compare band in lane 6 with that in lane 7 and lower band in lane 8). This was expected, since the natural $t k$ genes of HSV-1(F) $\Delta 305$ and R7214, which was derived from HSV-1(F) $\Delta 305$, are truncated by approximately 500 bp relative to wild-type $t k$ genes $(3,29)$.

(iv) The Bam HI T fragment of the R7228 virus migrated at a position consistent with a size of $0.7 \mathrm{kbp}$ (Fig. 2, lane 4, band 2 ). Thus, it is truncated by approximately $1.8 \mathrm{kbp}$ relative to the corresponding bands of HSV-1(F) and HSV-1(F) $\Delta 305$, indicating that the deletion of the $\mathrm{U}_{\mathrm{L}} 21$ open reading frame was as designed.

(v) The BamHI Q fragment of R7228 viral DNA could not be differentiated with respect to its electrophoretic mobility from the Bam HI Q fragment of HSV-1(F) DNA. Inasmuch as the virus was selected for growth in HAT medium, we conclude that the $t k$ gene was rescued at its natural position.

(vi) The Bam HI Q and T fragments of R7242 viral DNA could not be differentiated with respect to size from the corresponding HSV-1(F) DNA fragment. Inasmuch as R7242 also shares with HSV-1(F) the characteristic wild-type plaque morphology on Vero cells, we conclude pending verification 


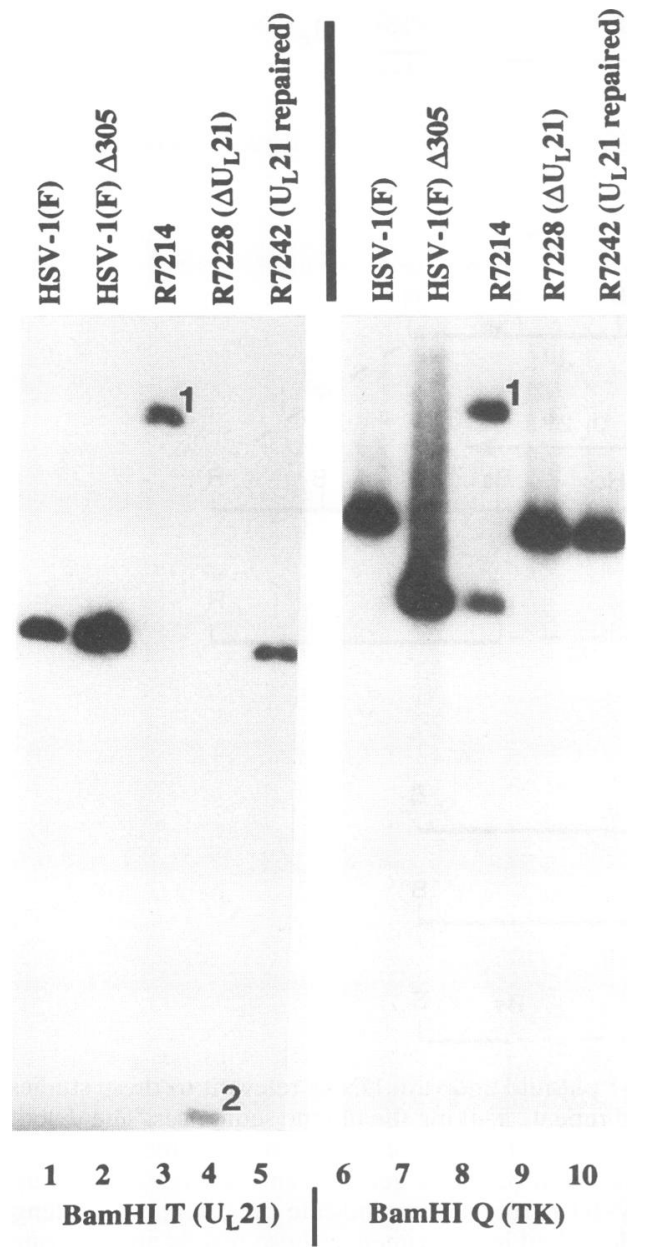

FIG. 2. Photograph of electrophoretically separated viral DNAs digested with Bam HI and hybridized with radiolabeled BamHI T and BamHI Q fragments of HSV-1(F). The indicated viral DNAs were purified, digested with $\mathrm{Bam} \mathrm{HI}$, electrophoretically separated on a $1 \%$ agarose gel, transferred to nitrocellulose, and hybridized with radiolabeled Bam $\mathrm{HI} T$ fragment containing $\mathrm{U}_{\mathrm{L}} 21$ sequences (lanes 1 to 5) or with the $B a m \mathrm{HI} \mathrm{Q}$ fragment containing $t k$ sequences (lanes 6 to 10 ). The origins of band 1 specific for R7214 DNA and band 2 specific for R7228 DNAs are indicated in the legend of Fig. 1. Band 1 hybridized with both BamHI T (lane 3) and BamHI Q (lane 8) probes. Band 2 arose as a consequence of the deletion of a portion of the $U_{L} 21$ sequences in the Bam HI T fragment.

with specific anti- $U_{L} 21$ antibody that the $U_{L} 21$ gene was rescued.

Replication of the $U_{L} 21$ deletion virus $R 7228$. In order to investigate the contribution of the $U_{L} 21$ gene to replication of HSV-1 in cell culture, replicate Vero or HEL cell monolayer cultures in $25-\mathrm{cm}^{2}$ cell culture flasks were infected with 5 PFU of HSV-1(F), R7228, or R7242 per cell. At various times after infection, the flasks were frozen at $-80^{\circ} \mathrm{C}$. At the completion of the experiment, the frozen-thawed cell lysates were sonicated and titrated in Vero cell monolayer cultures. As shown in Fig. 3, the replication patterns of HSV-1(F), R7228, and R7242 viruses in Vero cell monolayer cultures were virtually identical (Fig. 3, left panel). In HEL cell cultures, cells infected with the $\Delta \mathrm{U}_{\mathrm{L}} 21$ (R7228) virus yielded approximately three- to fivefold less virus than HSV-1(F)- or R7242-infected cells. Inasmuch as the $\mathrm{R} 7242$ virus in which the $\mathrm{U}_{\mathrm{L}} 21$ gene was restored repli-

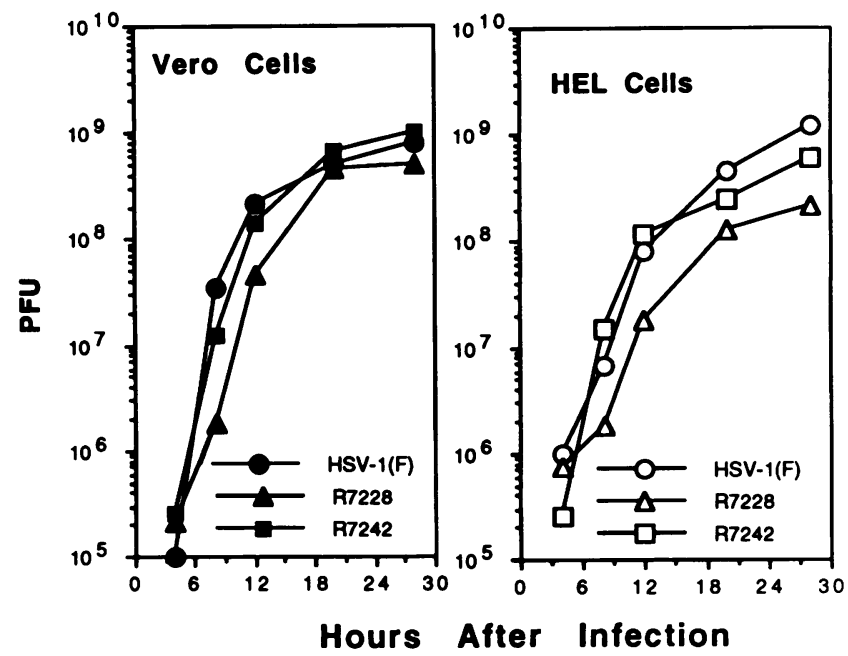

FIG. 3. Growth of wild-type and recombinant viruses in Vero and HEL cells. Monolayers of Vero (left panel) and HEL cells (right panel) maintained in $25-\mathrm{cm}^{2}$ dishes were infected with HSV-1(F), the $\mathrm{U}_{\mathrm{L}} 21$ deletion virus $\mathrm{R} 7228$, or the $\mathrm{U}_{\mathrm{L}} 21$ restored virus $\mathrm{R} 7242$. Cells and medium were frozen at various times after infection. Total infectious virus shown per $25-\mathrm{cm}^{2}$ flask was determined by plaque assays on Vero cell monolayer cultures.

cated like the wild-type virus, the slight decrease in $\mathrm{R} 7228$ virus yields in HEL was due to the deletion in the $U_{L} 21$ gene.

Production of antiserum directed against a purified $U_{L} 21$ GST bacterial fusion protein. The procedure used to obtain polyclonal antibody to the product of the $U_{L} 21$ gene was as follows. In the first step, we inserted the epitope from glycoprotein $\mathrm{B}$ of CMV reactive with the monoclonal antibody CH28-2 into the $U_{\mathrm{L}} 21$ open reading frame and cloned the majority of the tagged $\mathrm{U}_{\mathrm{L}} 21$ coding sequences in frame with the gene encoding GST. This procedure allowed a quick and reliable verification that in the bacterial cell lysates the product of the fusion of the $U_{L} 21$ coding sequences was in frame with the GST-coding domain by virtue of reactivity with the monoclonal antibody $\mathrm{CH} 28-2$ directed against the inserted tag. To identify bacterial clones that produced the expected fusion protein, log-phase bacterial cultures were grown in the presence or absence of $0.3 \mathrm{mM}$ isopropyl- $\beta$-D-thiogalactopyranoside (IPTG) for $2 \mathrm{~h}$. IPTG was expected to derepress the bacterial lac promoter and thus induce expression of the $\mathrm{U}_{\mathrm{L}}$ 21-GST fusion protein. Lysates from these cells were then electrophoretically separated on an SDS-polyacrylamide gel and were transferred electrically to a nitrocellulose sheet. The nitrocellulose sheet was then probed with the monoclonal antibody $\mathrm{CH} 28-2$. The results (Fig. 4) showed that whereas the lysates of uninduced cultures did not react with the monoclonal antibody, the lysates of bacteria induced by IPTG contained a major band with an apparent $M_{\mathrm{r}}$ of 66,000 which reacted with the $\mathrm{CH} 28-2$ monoclonal antibody. In addition, several more rapidly migrating bands also reacted with the monoclonal antibody. The more rapidly migrating bands vary in size and number from experiment to experiment.

We tentatively concluded from these results that the $U_{L} 21$ portion was in frame with the GST residues of the fusion protein inasmuch as the reactivity with the tag inserted into the $\mathrm{U}_{\mathrm{L}} 21$ gene was maintained and the size of the largest reactive band was as predicted from the GST and $U_{L} 21$ gene sequences. That the proper frame was maintained was confirmed by sequencing DNA encoding the junction of the GST and 


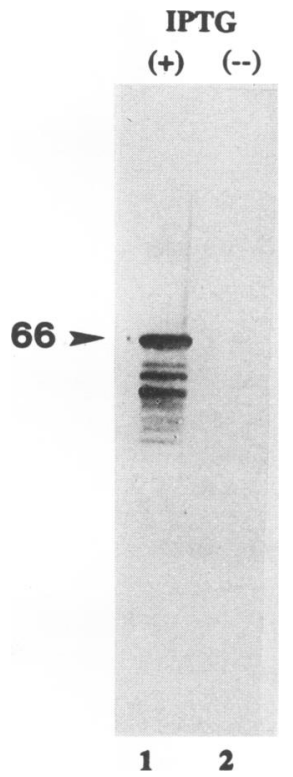

FIG. 4. Photograph of immunoblot of electrophoretically separated bacterial cell lysates probed with anti-CMV monoclonal antibody. A plasmid containing epitopically tagged $U_{L} 21$ sequences fused to GST under the control of the lac repressor was transformed into bacteria. The bacteria were grown in the presence $(+$, lane 1$)$ or absence ( - , lane 2$)$ of IPTG. The lysates were solubilized in SDS, separated on a denaturing polyacrylamide gel, electrically transferred to a nitrocellulose sheet, and reacted with the monoclonal antibody directed against the inserted CMV epitopic tag. The bound antibody was detected with goat anti-mouse IgG conjugated to alkaline phosphatase and then by fixation of alkaline phosphatase substrate. The apparent $M_{\mathrm{r}}$ (in thousands) of the major band is indicated.

$\mathrm{U}_{\mathrm{L}} 21$ genes (not shown). The smaller polypeptide bands most likely represent proteolytic cleavage products.

The preparation of the fusion protein for immunization of rabbits was as described in Materials and Methods.

Characterization of the $U_{L} 21$ gene product. In order to determine the specificity of the anti- $U_{L} 21-G S T$ antiserum, HEp-2 cells were mock infected or were infected with HSV$1(F), R 7228$ lacking the $U_{L} 21$ open reading frame, or $R 7242$ containing the restored $U_{L} 21$ gene. Lysates of the infected cells were then separated on a $9.0 \%$ polyacrylamide gel and were transferred electrically to a nitrocellulose sheet. The sheet was then probed with a 1:1,000 dilution of the $U_{L} 21$ antiserum. As shown in Fig. 5, the rabbit polyclonal antibody reacted with a broad band in electrophoretically separated lysates of HSV-1(F)- or R7242-infected cells. The electrophoretic mobility of this band was compatible with an apparent $M_{\mathrm{r}}$ ranging from 62,000 to 64,000 . In some lysates, the antibody also reacted with a very faint band with the electrophoretic mobility corresponding to an $M_{\mathrm{r}}$ of 60,000 in electrophoretically separated lysates of mock-infected cells (Fig. 5).

In order to determine whether the $U_{L} 21$ protein was expressed during the course of infection as a late gene, cells were infected with HSV-1(F) and the $U_{L} 21$ deletion virus $R 7228$ at 5.0 PFU per cell in the presence or absence of phosphonoacetic acid. In the presence of phosphonoacetic acid, the expression of the band with an apparent $M_{\mathrm{r}}$ of 62,000 to 64,000 was significantly reduced but not eliminated (Fig. 6). This indicated that the $U_{L} 21$ protein was expressed as a $\gamma_{1}$ gene, i.e., its expression was diminished but not abolished by inhibitors of DNA synthesis.

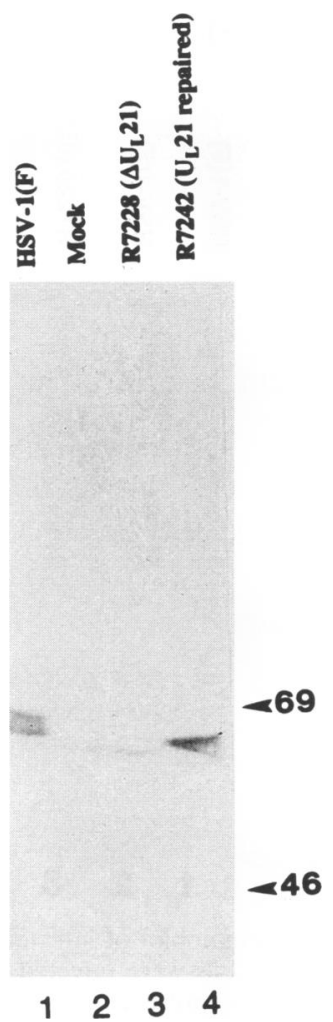

FIG. 5. Photograph of an immunoblot of mock-infected and infected cell lysates probed with rabbit polyclonal antiserum directed against the $U_{L} 21-G S T$ fusion protein. HEp-2 cells were either mock infected (Mock, lane 2) or were infected with the indicated viruses at 5.0 PFU per cell. At $18 \mathrm{~h}$ after infection, the cells were lysed and the proteins were separated on a denaturing polyacrylamide gel, transferred to nitrocellulose, and probed with the rabbit antiserum directed against the $U_{L} 21-G S T$ fusion protein. The positions of the migration of protein standards with $M_{\mathrm{r}} \mathrm{s} 69,000(69)$ and 46,000 (46) are indicated.

$U_{L} 21$ is a virion component. In order to determine whether the $U_{L} 21$ protein was associated with virions, purified HSV$1(F)$ and $R 7228$ virions from infected HEp-2 cells were solubilized, electrophoretically separated in a denaturing polyacrylamide gel, transferred to a nitrocellulose sheet, and probed with the anti- $U_{L} 21$ rabbit polyclonal antibody. As shown in Fig. 7, the $U_{L} 21$ protein was present in the virion preparation as a protein with an apparent $M_{\mathrm{r}}$ of 62,000 to 64,000 .

Localization of $U_{L} 21$ protein. HEp-2 cells were grown in LabTek (Nunc) cell culture dishes and were infected with the $\mathrm{gE}^{-} \mathrm{R7202}$ virus to avoid the strong reactivity of rabbit immunoglobulin $\mathrm{G}$ (IgG) with the Fc receptor specified by $\mathrm{gE}$. The cells were fixed in cold methanol at $18 \mathrm{~h}$ after infection and reacted with a 1:100 dilution of the $U_{L} 21$-specific serum and then fluorescein-conjugated goat anti-rabbit IgG. The cells were washed extensively, air dried, and viewed under a fluorescence microscope. As shown in Fig. 8B and C, in infected cells the $U_{L} 21$ protein localized primarily in the cytoplasm. In most infected cells the fluorescence staining was punctate and dispersed in the cytoplasm. In many cells the fluorescence was brightest within a perinuclear region of the infected cells. Occasional cells also contained diffuse fluorescence over infected cell nuclei. 


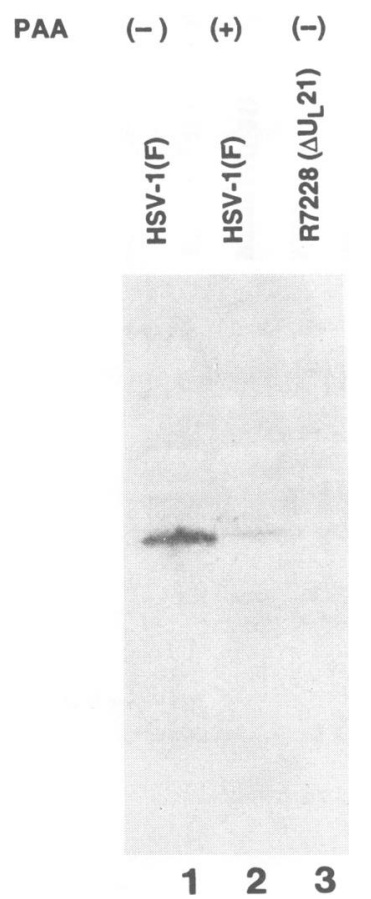

FIG. 6. Photograph of immunoblot of infected cell lysates probed with anti- $\mathrm{U}_{\mathrm{L}} 21-\mathrm{GST}$. HEp-2 cells were infected at 5.0 PFU per cell with the indicated viruses in the presence $(+$, lane 2$)$ or absence $(-$, lanes 1 and 3 ) of phosphonoacetic acid (PAA; $300 \mu \mathrm{g} / \mathrm{ml}$ of medium) The cells were harvested $24 \mathrm{~h}$ after infection, solubilized, electrophoretically separated in a denaturing polyacrylamide gel, transferred to nitrocellulose, and reacted with the rabbit polyclonal antiserum directed against the $\mathrm{U}_{\mathrm{L}} 21-\mathrm{GST}$ fusion protein.

\section{DISCUSSION}

In this report we showed the following. (i) The $U_{L} 21$ gene is dispensable for replication in cell culture. In the cell lines tested, no phenotype attributable to $U_{L} 21$ was apparent, although a three- to fivefold decrease in titer of primary human fibroblast (HEL) cells was observed. Thus, the $U_{L} 21$ gene joins a large number of HSV-1 genes dispensable for replication in cells in culture. The requirement for this gene in experimental animal systems is not yet known. We should note, however, that none of the genes dispensable for replication in cells in culture is truly dispensable in nature, since with few exceptions, none of the deletion mutants tested extensively in experimental animal systems is equivalent to wild-type virus with respect to replication or virulence. Moreover, mutants with deletion in these genes have not been isolated from infected humans.

$\mathrm{U}_{\mathrm{L}} 21$ thus appears to be an addition to the list of $\mathrm{U}_{\mathrm{L}}$ genes dispensable for viral replication in at least one primary cell strain and one continuous cell line.

(ii) The HSV-1 $\mathrm{U}_{\mathrm{L}} 21$ gene is a virion component. Results reported elsewhere suggested that the $U_{L} 21$ homolog of PRV was a component of the capsid and greatly facilitated DNA cleavage and packaging in cultured cells (11). Since the HSV-1 $\Delta \mathrm{U}_{\mathrm{L}} 21$ virus reported here replicated with kinetics identical to those of wild-type virus and since in the cells tested no evidence of defects in cleavage and packaging of HSV-1 viral DNA was found (data not shown), the apparent discrepancy may reflect the possibilities that the respective genes have different functions in the two viral systems, the PRV insertion mutant contained an additional mutation other than the

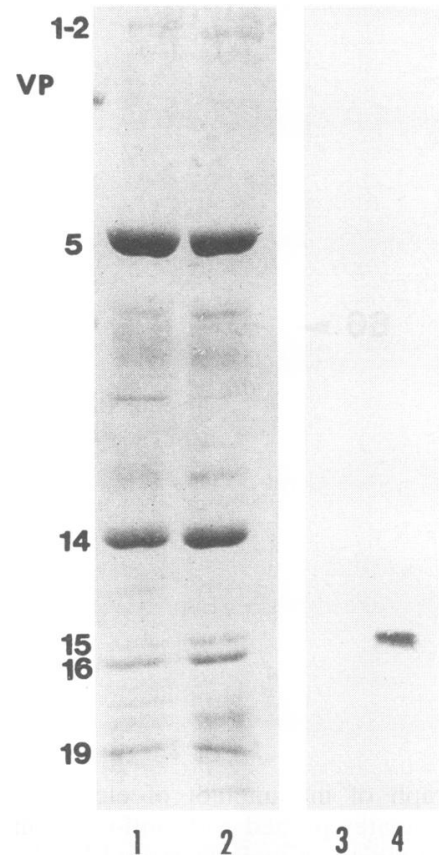

FIG. 7. Photograph of Coomassie blue-stained polyacrylamide gel and immunoblot of electrophoretically separated virion proteins. Proteins obtained from virions purified from HSV-1(F)-infected cells (lanes 2 and 4) or R7228-infected cells (lanes 1 and 3) were electrophoretically separated on a $9.0 \%$ denaturing polyacrylamide gel and were stained with Coomassie blue (lanes 1 and 2) or transferred to nitrocellulose and probed with the anti- $\mathrm{U}_{\mathrm{L}} 21-\mathrm{GST}$ polyclonal antibody diluted 1:10,000. Lanes 1 and 2 contain approximately 20-fold more total protein than lanes 3 and 4 .

truncation of the $U_{L} 21$ homolog, and the truncated PRV $U_{L} 21$ protein interfered with normal PRV DNA packaging.

Parenthetically, it is unlikely that the HSV-1 $\mathrm{U}_{\mathrm{L}} 21$ protein is a structural component of the capsid, inasmuch as all capsid proteins identified so far appear to be required for formation of infectious progeny and the genes encoding the seven HSV-1 capsid proteins have been identified $(9,10,19,26,33,41)$. In addition, the HSV-1 $\mathrm{U}_{\mathrm{L}} 21$ protein localized primarily in the perinuclear space and in the cytoplasm of infected cells, whereas capsid assembly takes place in the nucleus.

(iii) The $U_{L} 21$ open reading frame predicts that the $U_{L} 21$ protein has an $M_{\mathrm{r}}$ of 57,638 (23). We determined that the HSV-1-encoded $\mathrm{U}_{\mathrm{L}} 21$ protein present in the infected cells or associated with virions migrated with an apparent $M_{\mathrm{r}}$ of 62,000 to 64,000 . The broad appearance of the $U_{L} 21$ protein band in denaturing polyacrylamide gels suggests that the protein is modified posttranslationally, but the modification, if any, has not been identified. It is noteworthy that the antiserum directed against the PRV $U_{L} 21$ protein homolog immunoprecipitated proteins which formed two sharp bands with apparent $M_{\mathrm{r}} \mathrm{s}$ of 62,000 and 41,000. Among the possible explanations for the differences in the behavior of the PRV and HSV-1 homologs are that the homologous HSV-1 protein with an $M_{\mathrm{r}}$ of 41,000 did not react with the rabbit polyclonal antibody, the respective proteins are processed differently, and the protein with an apparent $M_{\mathrm{r}}$ of 41,000 is not a product of the PRV $\mathrm{U}_{\mathrm{L}} 21$ gene but is derived from another gene and was coimmunoprecipitated with the anti-PRV $U_{L} 21$ antiserum. Parenthetically, the HSV-1 $U_{L} 21$ open reading frame does not predict the existence of a glycine-aspartic acid-rich region speculated 

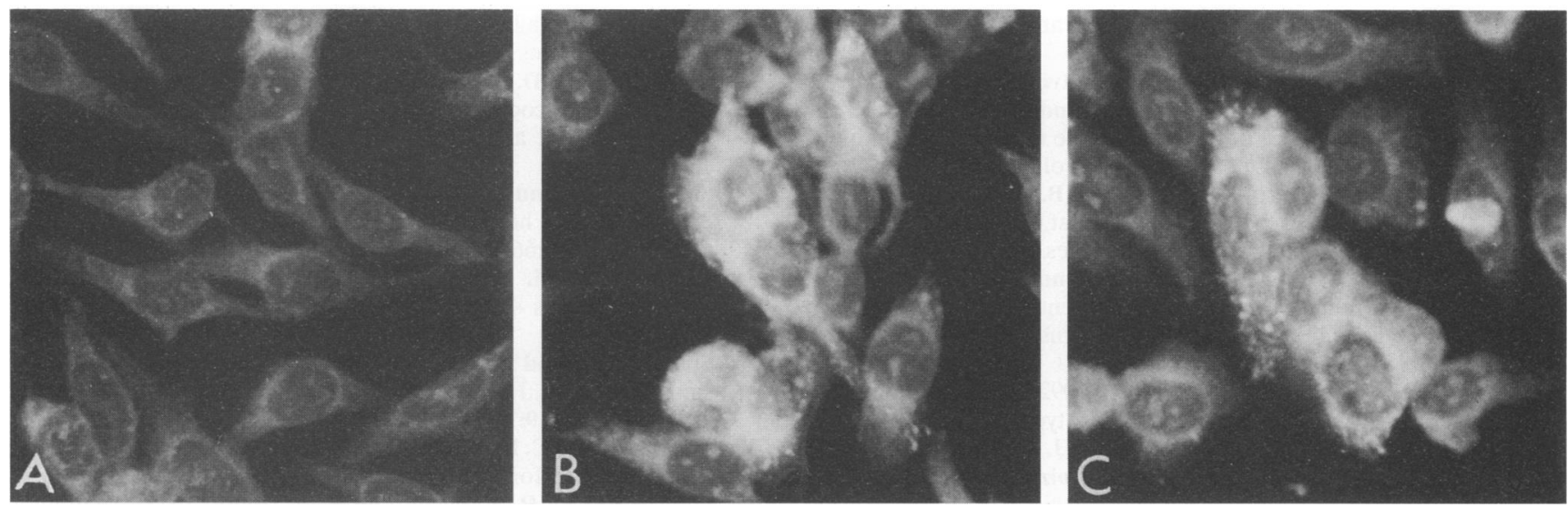

FIG. 8. Photograph of HEp-2 cells either mock infected or infected with the $\mathrm{gE}^{-} \mathrm{R} 7202$ virus, probed with rabbit polyclonal antibody directed against the $U_{L}$ 21-GST fusion protein, and reacted with fluorescein-conjugated goat anti-rabbit IgG. Cells were mock infected (A) or infected with the R7202 virus (B and C). Eighteen hours after infection, the cells were fixed in cold methanol and were probed with the rabbit antiserum and then reacted with fluorescein-conjugated goat anti-rabbit IgG.

to serve as a substrate for proteolytic cleavage of the PRV $\mathrm{U}_{\mathrm{L}} 21$ homolog.

\section{ACKNOWLEDGMENTS}

We thank Suzanna Rudofsky for technical assistance.

The studies at the University of Chicago were aided by grants from the National Cancer Institute (CA47451) and the National Institute for Allergy and Infectious Diseases (AI124009), the United States Public Health Service, and an unrestricted grant from the BristolMyers Squibb Program in Infectious Diseases. A.H.K. was supported, in part, by the Ministry of Science, Education, and Culture, Japan.

\section{REFERENCES}

1. Ackermann, M., J. Chou, M. Sarmiento, R. A. Lerner, and B. Roizman. 1986. Identification by antibody to a synthetic peptide of a protein specified by a diploid gene located in the terminal repeats of the $\mathrm{L}$ component of herpes simplex virus genome. J. Virol. 58:843-850.

2. Baines, J. D., and B. Roizman. 1991. The open reading frames UL3, UL4, UL10, and UL16 are dispensable for the growth of herpes simplex virus 1 in cell culture. J. Virol. 65:938-944.

3. Baines, J. D., and B. Roizman. 1992. The $U_{L} 11$ gene of herpes simplex virus 1 encodes a function that facilitates nucleocapsid envelopment and egress from cells. J. Virol. 66:5168-5174.

4. Baines, J. D., and B. Roizman. 1993. The $U_{L} 10$ gene of herpes simplex virus 1 encodes a novel glycoprotein, gM, which is present in the virion and in the plasma membrane of infected cells. J. Virol. 67:1441-1452.

5. Baines, J. D., P. L. Ward, G. Campadelli-Fiume, and B. Roizman. 1991. The $U_{L} 20$ gene of herpes simplex virus 1 encodes a function necessary for viral egress. J. Virol. 65:6414-6424.

6. Barker, D., and B. Roizman. 1992. The unique sequence of the herpes simplex virus $1 \mathrm{~L}$ component contains an additional open reading frame designated $U_{L} 49.5$. J. Virol. 66:562-572.

7. Barker, D. E., and B. Roizman. 1990. Identification of 3 genes nonessential for growth in cell culture near the right terminus of the unique sequences of long component of herpes simplex virus 1. Virology 177:684-691.

8. Basgoz, N., I. Qadri, D. Navarro, A. Sears, E. Lennette, J. Youngbloom, and L. Pereira. 1992. The aminoterminus of human cytomegalovirus glycoprotein B contains epitopes that vary among strains. J. Gen. Virol. 73:983-988.

9. Costa, R. H., G. Cohen, R. Eisenberg, D. Long, and E. Wagner. 1984. Direct demonstration that the abundant 6-kilobase herpes simplex virus type 1 mRNA mapping between 0.23 and 0.27 map units encodes the major capsid protein VP5. J. Virol. 49:287-292.
10. Davison, M. D., F. J. Rixon, and A. J. Davison. 1992. Identification of genes encoding two capsid proteins (VP24 and VP26) of herpes simplex type 1. J. Gen. Virol. 73:2709-2713.

11. de Wind, N., F. Wagenaar, J. Pol, T. Kimman, and A. Berns. 1992. The pseudorabies virus homolog of the herpes simplex UL21 gene product is a capsid protein which is involved in capsid maturation. J. Virol. 66:7096-7103.

12. Ejercito, P. M., E. D. Kieff, and B. Roizman. 1968. Characterization of herpes simplex virus strains differing in their effects on social behavior of infected cells. J. Gen. Virol. 2:357-364.

13. Fenwick, M. L., and R. D. Everett. 1990. Inactivation of the shutoff gene (UL41) of herpes simplex virus types 1 and 2. J. Gen. Virol. 71:2961-2967.

14. Fisher, F. B., and V. G. Preston. 1986. Isolation and characterization of herpes simplex virus type 1 mutants which fail to induce dUTPase activity. Virology 148:190-197.

15. Georgeopoulou, U., A. Michaelidou, B. Roizman, and P. Mavromara-Nazos. 1993. Identification of a new transcriptional unit that yields a gene product within the unique sequences of the short component of the herpes simplex virus 1 genome. J. Virol. 67:3961-3968.

16. Goldstein, D. J., and S. K. Weller. 1988. Herpes simplex virus type 1-induced ribonucleotide reductase activity is dispensable for virus growth and DNA synthesis: isolation and characterization of an ICP6 lacZ insertion mutant. J. Virol. 62:196-205.

17. Hayward, G. S., R. J. Jacob, S. C. Wadsworth, and B. Roizman. 1975. Anatomy of herpes simplex virus DNA: evidence for four populations of molecules that differ in the relative orientations of their long and short segments. Proc. Natl. Acad. Sci. USA 72:4243-4247.

18. Heine, J. W., R. W. Honess, E. Cassai, and B. Roizman. 1974. Proteins specified by herpes simplex virus. XII. The virion polypeptides of type 1 strains. J. Virol. 14:640-651.

19. Liu, F., and B. Roizman. 1991. The promoter, transcriptional unit, and coding sequences of herpes simplex virus 1 family 35 proteins are contained within and in frame with the UL26 open reading frame. J. Virol. 65:206-212.

20. Longnecker, R., and B. Roizman. 1986. Generation of an inverting herpes simplex virus 1 mutant lacking the L-S junction a sequences, an origin of DNA synthesis, and several genes including those specifying glycoprotein $\mathrm{E}$ and the $\alpha 47$ gene. J. Virol. 58:583-591.

21. MacLean, C. A., A. Dolan, F. E. Jamieson, and D. J. McGeoch 1992. The myristylated virion proteins of herpes simplex virus type 1: investigation of their role in the virus life cycle. J. Gen. Virol. 73:539-547.

22. MacLean, C. A., S. Efstathiou, M. L. Elliott, F. E. Jamieson, and D. J. McGeoch. 1991. Investigation of herpes simplex virus type 1 
genes encoding multiply inserted membrane proteins. J. Gen. Virol. 72:897-906.

23. McGeoch, D. J., M. A. Dalrymple, A. J. Davison, A. Dolan, M. C. Frame, D. McNab, L. J. Perry, J. E. Scott, and P. Taylor. 1988. The complete DNA sequence of the long unique region in the genome of herpes simplex virus type 1. J. Gen. Virol. 69:1531-1574.

24. McGeoch, D. J., A. Dolan, S. Donald, and B. H. K. Brauer. 1986. Complete DNA sequence of the short repeat region in the genome of herpes simplex type 1. Nucleic Acids Res. 14:1727-1745.

25. McGeoch, D. J., A. Dolan, S. Donald, and F. J. Rixon. 1985. Sequence determination and genetic content of the short unique region in the genome of herpes simplex virus type 1. J. Mol. Biol. 181:1-13.

26. McNabb, D. S., and R. J. Courtney. 1992. Identification and characterization of the herpes simplex type 1 virion protein encoded by the UL35 open reading frame. J. Virol. 66:2653-2663.

27. Meignier, B., R. Longnecker, and B. Roizman. 1988. In vivo behavior of genetically engineered herpes simplex viruses R7017 and R7020: construction and evaluation in rodents. J. Infect. Dis. 158:602-614.

28. Mullaney, J., H. W. Moss, and D. J. McGeoch. 1989. Gene UL2 of herpes simplex virus type 1 encodes a uracil-DNA glycosylase. J. Gen. Virol. 70:4490-4540.

29. Post, L. E., S. Mackem, and B. Roizman. 1981. Regulation of $\alpha$ genes of herpes simplex virus: expression of chimeric genes produced by fusion of thymidine kinase with $\alpha$ gene promoters. Cell 24:555-565.

30. Post, L. E., and B. Roizman. 1981. A generalized technique for deletion of specific genes in large genomes: $\alpha$ gene 22 of herpes simplex virus is not essential for growth. Cell 25:227-232.

31. Purves, F. C., and B. Roizman. 1992. The $U_{L} 13$ gene of herpes simplex virus 1 encodes the functions for posttranslational processing associated with phosphorylation of the regulatory protein a22. Proc. Natl. Acad. Sci. USA 89:7310-7314.

32. Read, G. S., and N. Frenkel. 1983. Herpes simplex virus mutants defective in the virion-associated host shutoff of host polypeptide synthesis and exhibiting abnormal synthesis of $\alpha$ (immediate early) viral polypeptides. J. Virol. 46:498-512.

33. Rixon, F. J., M. D. Davison, and A. J. Davison. 1990. Identification of the genes encoding two capsid proteins of herpes simplex virus type 1 by direct amino acid sequencing. J. Gen. Virol. 71:12111214.

34. Sheldrick, P., and N. Berthelot. 1975. Inverted repetitions in the chromosome of herpes simplex virus. Cold Spring Harbor Symp. Quant. Biol. 39:667-678.

35. Southern, E. M. 1975. Detection of specific sequences among DNA fragments separated by gel electrophoresis. J. Mol. Biol. 98:503-517.

36. Spear, P. G., and B. Roizman. 1972. Proteins specified by herpes simplex virus. V. Purification and structural proteins of the herpes virion. J. Virol. 9:143-159.

37. Visalli, R., and C. R. Brandt. 1991. The HSV-1 UL45 gene product is not required for growth in Vero cells. Virology 185:419-423.

38. Wadsworth, S., R. J. Jacob, and B. Roizman. 1975. Anatomy of herpes simplex virus DNA. II. Size, composition, and arrangement of inverted terminal repetitions. J. Virol. 15:1487-1497.

39. Walboomers, J. M., and J. Ter Schagget. 1976. A new method for the isolation of herpes simplex virus type 2 DNA. Virology 74:256-258.

40. Weller, S. K., M. R. Seghatoleslami, L. Shao, D. Rowse, and E. P. Carmichael. 1990. The herpes simplex virus type 1 alkaline nuclease is not essential for viral DNA synthesis: isolation and characterization of a lacZ insertion mutant. J. Gen. Virol. 71: 2941-2952.

41. Yei, S., S. I. Chowdury, B. M. Bhat, A. J. Conley, W. S. Wold, and W. Batterson. 1990. Identification and characterization of the herpes simplex virus type 2 gene encoding the essential capsid protein ICP32/VP19c. J. Virol. 64:1124-1134.

42. Zhang, Y., D. A. Sirko, and J. L. C. McKnight. 1991. Role of herpes simplex virus type 1 UL46 and UL47 in $\alpha$ TIF-mediated induction: characterization of three viral deletion mutants. J. Virol. 65:829-841. 